\title{
Fluctuations in quantum devices
}

\author{
H.Haken \\ Institute for Theoretical Physics, Center of Synergetics, \\ University of Stuttgart, \\ Pfaffenwaldring 57/4, D-70550 Stuttgart, Germany
}

Received March 16, 2004

\begin{abstract}
Logical gates can be formalized by Boolean algebra whose elementary operations can be realized by devices that employ the interactions of macroscopic numbers of elementary excitations such as electrons, holes, photons etc. With increasing miniaturization to the nano scale and below, quantum fluctuations become important and can no longer be ignored. Based on Heisenberg equations of motion for the creation and annihilation operators of elementary excitations, I determine the noise sources of composite quantum systems.
\end{abstract}

Key words: quantum fluctuations, elementary excitations, Heisenberg equations of motion, logical devices, noise in composite quantum systems

PACS: 05.40.-a, 05.30.-d

\section{Introduction}

Since my contribution is dedicated to the memory of my friend Yuri Klimontovich, I wish to start with a few personal remarks. I met Yuri for the first time in 1963 when I was invited to attend and to give a paper at the All Union Conference on Solid State Physics in Moscow. This was my first visit to the Soviet Union and I was overwhelmed by the warm reception I received there after such a terrible war. I gave a lecture on laser theory that met special interest by Yuri. He read my preprint and we had a very stimulating discussion on it. I was impressed by the great warmth of Yuri and we became friends immediately. Later I had again and again opportunities to meet Yuri at various conferences in the Soviet Union. I especially remember a conference in Pushchino on the field of synergetics. I was delighted to see the reception of our research and I realized how much contributions had been done previously in Russia to this field. I was also very pleased that Yuri and his colleagues initiated a permanent and very lively seminar on synergetics at the Lomonossov University in Moscow. I tried of course to invite Yuri to my own institute in Stuttgart because I had some funds there. For the well known reasons, however, he was not able to come until the new situation occurred. Since then I 
was very happy to have him several times in Stuttgart and we enjoyed both our scientific discussions and life very much. I was deeply impressed by the profound ideas of Yuri, especially in the field of non-equilibrium physics [1] that was a field of our common interest and I profited again and again by his deep insights, e.g. on his by now famous S-theorem. The last time I met Yuri was in Berlin at Werner Ebeling's institute just a few months before his untimely death. Yuri was such a warm hearted and noble personality and I surely will not forget him.

My paper is motivated by the progress made in information technology and here again especially in nanotechnology. So far information processing is based on logical elements that use large numbers of elementary excitations such as electrons so that quantum fluctuations can be ignored, at least in many cases. With increasing miniaturization, however, the quantum domain becomes important. Therefore my contribution tries to show how quantum fluctuations can be calculated in a unique way for all kinds of elementary excitations and their interactions that may be used in logical devices.

In my contribution I consider logical devices or, in other words, logical gates. As is well known logical operations can be formalized by a Boolean algebra which contains in particular the operations "and", "or", and the "exclusive or". Their corresponding truth tables can be realized by specific dynamical systems of which we write down the typical equations

$$
\begin{aligned}
\text { "and" : } & \frac{\mathrm{d} n_{\mathrm{r}}}{\mathrm{d} t}=g n_{1} n_{2}, \quad n \geqslant 0, \\
\text { "or" : } \quad \frac{\mathrm{d} n_{\mathrm{r}}}{\mathrm{d} t}=g\left(n_{1}+n_{2}\right), \quad n \geqslant 0, & \frac{\mathrm{d} x}{\mathrm{~d} t}=-3 y z-\left(x^{2}+y^{2}+z^{2}\right) x, \\
\text { "exclusiveor" : } \quad & \frac{\mathrm{d} y}{\mathrm{~d} t}=-3 x z-\left(x^{2}+y^{2}+z^{2}\right) y,
\end{aligned}
$$

and

$$
\frac{\mathrm{d} z}{\mathrm{~d} t}=-3 x y-\left(x^{2}+y^{2}+z^{2}\right) z .
$$

These equations must be solved by means of an initial value problem where the transients provide the solutions to the corresponding truth tables. In the cases (1) and (2) the reader may easily verify the validity of the corresponding truth tables while with respect to the equations (3)-(5) I refer the reader to my book "Synergetic computers and cognition" [2]. When we want to realize these abstract equations by means of devices where e.g. the quantities $n$ refer to currents or charge densities, we must take care of their coupling to the surrounding which may be represented by heatbaths. The elimination of the heatbath variables then leads to equations that represent losses as well. Thus for instance equation (1) must be replaced by equation

$$
\frac{\mathrm{d} n_{\mathrm{r}}}{\mathrm{d} t}=g n_{1} n_{2}-k n_{\mathrm{r}} .
$$

With increasing miniaturization going to the nano scale and especially to still smaller dimensions the processes must be treated from a quantum mechanical point 
of view. Thus the obvious question arises how to translate the above equations into quantum mechanics. Here we must be very careful because there are different ways depending on the experimental setup. In other words, we must take care of the quantum mechanical preknowledge we have or, in other words, we must take care of the way the initial states are prepared. Our considerations will apply basically to all sorts of elementary excitations in solid state physics such as electrons, holes, excitons, plasmons, polaritons, spin waves as well as to photons. In the first approach I am proposing the replacement

$$
n_{\mathrm{r}}, n_{1}, n_{2} \rightarrow \text { quantum amplitudes } b, a, c,
$$

where $b, a, c$ will become quantum mechanical operators. In a more detailed description, these operators must be equipped with indices, e.g. wave-vectors. To bring out the essentials, we drop such indices here. The interaction term in the case that will give rise to equation (1) can be described by a Hamiltonian of the form

$$
H=\hbar g\left(b^{+} a c+c^{+} a^{+} b\right)
$$

where $\hbar$ is Planck's constant divided by $2 \pi$ and $g$ is a coupling constant. The daggers indicate creation operators. Operators without daggers are annihilation operators. In order to facilitate my approach I will consider in the present case a subspace spanned by the vectors

$$
a^{+} c^{+} \phi_{0} \text { and } b^{+} \phi_{0}
$$

where $\phi_{0}$ is the vacuum state. In order to come as close as possible to the description in the terminology of a dynamical system I consider Heisenberg equations of motion. As is well known, for any operator $\Omega$ the Heisenberg equations can be derived by means of the Hamiltonian in the form

$$
\frac{\mathrm{d} \Omega}{\mathrm{d} t}=\frac{\mathrm{i}}{\hbar}[H, \Omega]
$$

where the square bracket indicates the commutator

$$
[H, \Omega]=H \Omega-\Omega H
$$

Thus the Heisenberg equation for the annihilation operator $b$ reads

$$
\frac{\mathrm{d} b}{\mathrm{~d} t}=\mathrm{i}\left[g\left(b^{+} a c+c^{+} a^{+} b\right), b\right]
$$

or because of commutation relations

$$
\frac{\mathrm{d} b}{\mathrm{~d} t}=\mathrm{i} g a c\left[b^{+}, b\right] .
$$

Using the explicit commutation relation

$$
\left[b^{+}, b\right]=-1
$$


we eventually arrive at

$$
\frac{\mathrm{d} b}{\mathrm{~d} t}=-\mathrm{i} g a c .
$$

Similarly the Heisenberg equations for the operator product $a c$ read

$$
\frac{\mathrm{d}(a c)}{\mathrm{d} t}=\mathrm{i} g\left[\left(b^{+} a c+c^{+} a^{+} b, a c\right]\right.
$$

or

$$
\frac{\mathrm{d}(a c)}{\mathrm{d} t}=\mathrm{i} g b\left[c^{+} a^{+}, a c\right]=\mathrm{i} g b\left(c^{+} a^{+} a c-a c c^{+} a^{+}\right)
$$

which because of

$$
a a^{+}-a^{+} a=1
$$

can be cast into the form

$$
\frac{\mathrm{d}(a c)}{\mathrm{d} t}=\mathrm{i} g b\left(c^{+} a^{+} a c-\left(1+a^{+} a\right)\left(1+c^{+} c\right)\right)
$$

or in short

$$
\frac{\mathrm{d}(a c)}{\mathrm{d} t}=-\mathrm{i} g b\left(1+a^{+} a+c^{+} c\right)
$$

Having in mind that the operators act in the subspace (9) the equation (20) reduces to

$$
\frac{\mathrm{d}(a c)}{\mathrm{d} t}=-\mathrm{i} g b
$$

By a similar approach we obtain the relations

$$
\begin{aligned}
\frac{\mathrm{d}(b a c)}{\mathrm{d} t} & =\mathrm{i} g\left[b^{+} a c+c^{+} a^{+} b, b a c=\right]= \\
& =\mathrm{i} g a c\left[b^{+}, b\right]+\mathrm{i} g b\left[c^{+} a^{+}, a c\right]= \\
& =-\mathrm{i} g a c-\mathrm{i} g b \quad \text { in subspace! }
\end{aligned}
$$

So far we have not taken into account the interaction of our quantum system with heatbaths. Having eliminated them (which can be done in a model like fashion explicitly) we may take care of their effect using the damping constants so that the above equations are translated into

$$
\begin{aligned}
\frac{\mathrm{d} b}{\mathrm{~d} t} & =-\mathrm{i} g a c-\kappa b, \\
\frac{\mathrm{d}(a c)}{\mathrm{d} t} & =-\mathrm{i} g b-\gamma a c
\end{aligned}
$$

and

$$
\frac{\mathrm{d}(b a c)}{\mathrm{d} t}=-\mathrm{i} g a c-\mathrm{i} g b-\gamma^{\prime}(b a c),
$$

respectively. However, this phenomenological approach has a great drawback because, as one may easily show, it violates quantum mechanical consistency. As has 
been shown especially in the context of laser physics this violation of quantum mechanical consistency can be remedied by the introduction of quantum noise operators $\Gamma$ so that for instance equation (23) is transformed into

$$
\frac{\mathrm{d} b}{\mathrm{~d} t}=-\mathrm{i} g a c-\kappa b+\Gamma_{b}(t)
$$

For reasons that will become obvious herein below I reformulate the corresponding quantum mechanical equations using projection operators $P_{i j}$ according to the scheme:

$$
b \rightarrow P_{01}^{1} ; \quad a c=P_{0011}^{2} ; \quad b a c \rightarrow P_{01}^{1} P_{0011}^{2} .
$$

This transforms the equations (23) and (24) into

$$
\frac{\mathrm{d} P_{01}^{1}}{\mathrm{~d} t}=-\mathrm{i} g P_{0011}^{2}-\kappa P_{01}^{1}+\Gamma_{01}^{1}
$$

and

$$
\frac{\mathrm{d} P_{0011}^{2}}{\mathrm{~d} t}=-\mathrm{i} g P_{01}^{1}-\gamma P_{0011}^{2}+\Gamma_{0011}^{2}
$$

respectively, and using the notation

$$
P_{01}^{1} P_{0011}^{2}=P_{000,111}^{0}
$$

we can cast (25) into the form

$$
\frac{\mathrm{d} P_{000,111}^{0}}{\mathrm{~d} t}=-\mathrm{i} g P_{0011}^{2}-\mathrm{i} g P_{01}^{1}-\gamma^{\prime} P_{000,111}^{0}+\Gamma_{000,111}^{0} .
$$

The obvious question arises in which way we may determine the fluctuating forces $\Gamma_{01}^{1}, \Gamma_{0011}^{2}, \Gamma_{000,111}^{0}$. Before we turn our attention to the solution of this task, we discuss a second interpretation. Namely the initial state may also be prepared using the occupation numbers and we may consider an experimental setup in which only occupation numbers of elementary excitations are concerned. Thus our goal is to translate the equation

$$
\frac{\mathrm{d} n_{\mathrm{r}}}{\mathrm{d} t}=g n_{1} n_{2}
$$

correspondingly which we first consider without damping. In our model we also assume that

$$
\frac{\mathrm{d}\left(n_{1} n_{2}\right)}{\mathrm{d} t}=g n_{\mathrm{r}}
$$

holds. From (33) and (32) we easily derive

$$
\frac{\mathrm{d}\left(n_{\mathrm{r}} n_{1} n_{2}\right)}{\mathrm{d} t}=g n_{1}^{2} n_{2}^{2}+n_{\mathrm{r}}^{2} g
$$

Introducing losses we may supplement the equations correspondingly so that for instance we obtain

$$
\frac{\mathrm{d} n_{\mathrm{r}}}{\mathrm{d} t}=g n_{1} n_{2}-\kappa n_{\mathrm{r}}
$$


and

$$
\frac{\mathrm{d}\left(n_{1} n_{2}\right)}{\mathrm{d} t}=g n_{\mathrm{r}}-\gamma n_{1} n_{2}
$$

When we choose $\kappa=\gamma=g$, (35) and (36) lead to a conservation law. In order to translate these equations into quantum mechanics we introduce projection operators according to

$$
n_{\mathrm{r}} \rightarrow P_{11}^{1}, \quad n_{1} n_{2} \rightarrow P_{11,11}^{2}
$$

so that we now have to consider the equations

$$
\begin{aligned}
\frac{\mathrm{d} P_{11}^{1}}{\mathrm{~d} t} & =g P_{11,11}^{2}-\kappa P_{11}^{1}+\Gamma_{11}^{1}, \\
\frac{\mathrm{d} P_{11,11}^{2}}{\mathrm{~d} t} & =g P_{11}^{1}-\gamma P_{11,11}^{2}+\Gamma_{11,11}^{2},
\end{aligned}
$$

and

$$
\frac{\mathrm{d}\left(P_{11}^{1} P_{11,11}^{2}\right)}{\mathrm{d} t}=g P_{11,11}^{2} P_{11,11}^{2}+g P_{11}^{1} P_{11}^{1}-(\kappa+\gamma) P_{11}^{1} P_{11,11}^{2}+\Gamma_{111,111}^{0} .
$$

Making use of the property of projection operators (see also below for details)

$$
P_{11}^{1} P_{11}^{1}=P_{11}^{1}
$$

and of

$$
P_{11}^{1} P_{11,11}^{22}=P_{111,111}^{0}
$$

we may cast (40)into the form

$$
\frac{\mathrm{d} P_{111,111}^{0}}{\mathrm{~d} t}=g P_{11,11}^{2}+g P_{11}^{1}-(\kappa+\gamma) P_{111,111}^{0}+\Gamma_{111,111}^{0} .
$$

Again it remains our task to determine the fluctuating forces $\Gamma$ explicitly.

\section{Haken-Weidlich theorem $[3,4]$}

In the following we use the fundamental quantum mechanical property of projection operators

$$
P_{i j} P_{l k}=\delta_{j l} P_{i k}
$$

We denote quantum statistical averages by square brackets. We assume that for instance phenomenologically or partly phenomenologically and partly from first principles the following averaged equations are given

$$
\frac{\mathrm{d}\left\langle P_{i j}\right\rangle}{\mathrm{d} t}=\sum_{k l}\left\langle M_{i j, k l} P_{k l}\right\rangle
$$

where the elements $M$ do not depend on $P$, but may depend on variables of other quantum systems. As one may show the solutions to (45) do not obey the quantum 
mechanical consistency relations (44). To restore quantum mechanical consistency we introduce the equations

$$
\frac{\mathrm{d} P_{i j}}{\mathrm{~d} t}=\sum_{k l} M_{i j k l} P_{k l}+\Gamma_{i j}(t) .
$$

We assume that the averages vanish

$$
\left\langle\Gamma_{i j}(t)\right\rangle=0
$$

and that the fluctuating forces are $\delta$-correlated in time

$$
\left\langle\Gamma_{i j}(t) \Gamma_{k l}\left(t^{\prime}\right)\right\rangle=G_{i j, k l} \delta\left(t-t^{\prime}\right) .
$$

This is the only assumption to be made in the present context. In many cases it is fulfilled if for instance the reservoirs are broadband or the relaxation time of the fluctuating forces is short compared to that of all other processes in the system. The Haken-Weidlich theorem states that the strength of the fluctuating forces is uniquely determined by

$$
G_{i j, k l}=\sum_{m n}\left\langle\left(\delta_{j k} M_{i l, m n}-\delta_{n l} M_{i j, m k}-\delta_{m i} M_{k l, j n}\right) P_{m n}\right\rangle .
$$

As a comparison with the previous section shows, $M$ may be decomposed into

$$
M_{i j, k l}=M_{i j, k l}^{(1)}+M_{i j, k l}^{(2)},
$$

where

$$
M^{(1)} \text { stems from } \frac{\mathrm{i}}{\hbar}\left[H, P_{i j}\right] .
$$

As can be shown, the terms $M^{(1)}$ cancel each other so that it is sufficient to determine the strengths of the fluctuating forces by using $M^{(2)}$ instead of $M$ in (49).

\section{Composite quantum systems}

In the following I want to generalize the Haken-Weidlich theorem to composite quantum systems. This requires the introduction of the appropriate multiplication rules of projection operators. First we adopt the already known rule

$$
P_{i j}^{1} P_{k l}^{1}=\delta_{j k} P_{i l}^{1}
$$

where the upper index lrefers to the specific subsystem. Similarly we have

$$
P_{i j}^{2} P_{k l}^{2}=\delta_{j k} P_{i l}^{2}
$$

However, what is new is the relation for the composite system given by

$$
P_{i j}^{1} P_{k l}^{2}=P_{i k, j l}^{0}
$$


From (52)-(54) we may deduce the following multiplication rules:

$$
\begin{aligned}
P_{i j}^{1} P_{i^{\prime} k^{\prime}, j^{\prime} l^{\prime}}^{0} & \equiv P_{i j}^{1} P_{i^{\prime}, j^{\prime}}^{1} P_{k^{\prime} l^{\prime}}^{2}=\delta_{j i^{\prime}} P_{i k^{\prime}, j^{\prime} l^{\prime}}^{0}, \\
P_{i j}^{2} P_{i^{\prime} k^{\prime}, j^{\prime} l^{\prime}}^{0} & =P_{i j}^{2} P_{i^{\prime} j^{\prime}}^{1} P_{k^{\prime} l^{\prime}}^{2}=\delta_{j k^{\prime}} P_{i^{\prime} i, j^{\prime} l^{\prime}}^{0}, \\
P_{i j, k l}^{0} P_{i^{\prime} j^{\prime}, k^{\prime} l^{\prime}}^{0} & =P_{i k}^{1} P_{j l}^{2} P_{i^{\prime} k^{\prime}}^{1} P_{j^{\prime} l^{\prime}}^{2}=\delta_{k i^{\prime}} \delta_{l j} P_{i j k^{\prime} l^{\prime}}^{0} .
\end{aligned}
$$

For the following we need a concise notation so that we introduce the following abbreviation:

$$
\begin{aligned}
& P_{\sim}^{K, j} \underset{\sim}{K} \quad \text { for } \quad K=1, \quad \underset{\sim}{i}=1, \quad \underset{\sim}{j}=j, \\
& K=2, \quad \underset{\sim}{i}=1, \quad \underset{\sim}{j}=j, \\
& K=0, \quad \underset{\sim}{i}=i_{1} i_{2}, \quad \underset{\sim}{j}=j_{1} j_{2} .
\end{aligned}
$$

Having used it we can cast the relations (52)-(54) in the concise form

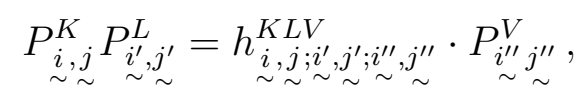

where for instance

$$
(K, L): 1,1, \quad h_{i j, i^{\prime} j^{\prime}, i^{\prime \prime} j^{\prime \prime}}^{111}=\delta_{j i^{\prime}} \delta_{i^{\prime \prime} i} \delta_{j^{\prime} j^{\prime \prime}}
$$

holds. The basic idea is now similar to that of section 4 . We assume that the averaged equations

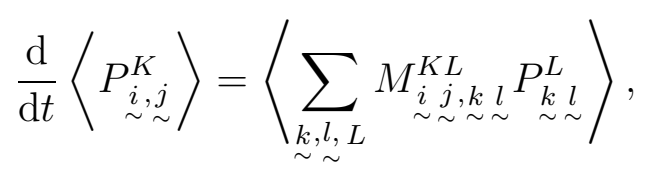

that may be either based on Hamiltonians and on phenomenologically added incoherent terms or containing only incoherent terms. We want to convert these equations into quantum mechanically consistent equations by adding fluctuating forces

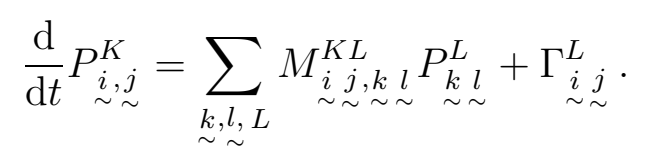

We lump the projection operators together to a state vector

$$
A=\left(\begin{array}{c}
P^{(1)} \\
P^{(2)} \\
P^{(0)}
\end{array}\right)
$$

that has to obey equations that we write in the form

$$
\frac{\mathrm{d} A}{\mathrm{~d} t}=M A+\Gamma .
$$


The formal solution of (64) reads

$$
A=\int^{t} G(t, \tau) \Gamma(\tau) \mathrm{d} \tau+A_{\mathrm{h}}
$$

where $G$ is the Green's function with the property

$$
G(t, t)=E
$$

and $A_{\mathrm{h}}$ a solution to the homogeneous equation (64). We consider

$$
\langle\widetilde{A} B A\rangle=\left\langle\left(\int^{t} \widetilde{\Gamma}(\tau) \widetilde{G}(t, \tau) \mathrm{d} \tau+\widetilde{A}_{\mathrm{h}}\right) B\left(\int^{t} G\left(t, \tau^{\prime}\right) \Gamma\left(\tau^{\prime}\right) \mathrm{d} \tau^{\prime}+A_{\mathrm{h}}\right)\right\rangle,
$$

where the tilde refers to the transposed matrix or transposed vector

$$
\widetilde{A}, \widetilde{B} \text {, etc. }
$$

We have introduced a matrix $B$ in the form

$$
B=\left(\begin{array}{lll}
B^{11} & B^{12} & B^{10} \\
B^{21} & B^{22} & B^{20} \\
B^{01} & B^{02} & B^{00}
\end{array}\right)
$$

where each submatrix is further labelled by means of indices,

$$
I=\underset{\sim}{i, j}
$$

where eventually we will choose only one non-vanishing element. Because of (59) we obtain for the l. h. side of (64)

$$
\left\langle P_{I}^{K} B_{I J}^{K L} P_{J}^{L}\right\rangle=B_{I J}^{K L}\left\langle P_{I}^{K} P_{J}^{L}\right\rangle=B_{I J}^{K L} h_{I, I^{\prime}, I^{\prime \prime}}^{K L V}\left\langle P_{I^{\prime \prime}}^{V}\right\rangle .
$$

Taking the derivative with respect of time, we then obtain for the 1 . h. side of (46) the relation

$$
\text { l.h.s } \frac{\mathrm{d}}{\mathrm{d} t}: \quad B_{I J}^{K L} h_{I, I^{\prime}, I^{\prime \prime}}^{K L V} \sum_{J, W}\left\langle M_{I^{\prime \prime} J}^{V W} P_{J}^{W}\right\rangle \text {. }
$$

After differentiation the r. h. side (67) contains the terms

$$
\begin{aligned}
\text { r.h.s } \frac{\mathrm{d}}{\mathrm{d} t}: & \left\langle\widetilde{\Gamma}(t) B\left(\int_{0}^{t} G(t, \tau) \Gamma(\tau) \mathrm{d} \tau+A_{\mathrm{h}}\right)\right\rangle, \\
& \left\langle\left(\int_{0}^{t} \widetilde{\Gamma}(\tau) \widetilde{G}(t, \tau) \mathrm{d} \tau+\widetilde{A}_{\mathrm{h}}\right) B \Gamma(\tau)\right\rangle, \\
& \langle\widetilde{A} \widetilde{M} B A\rangle, \\
& \langle\widetilde{A} \widetilde{B} M A\rangle .
\end{aligned}
$$


A simple analysis and using a single element of $B$ transforms (73) into

$$
B_{I J}^{K L} \frac{1}{2} G_{I J}^{K L}
$$

and the same expression results from (74). The expression (76) can easily be transformed into

$$
B_{I J}^{K L}\left\langle P_{I}^{K} \sum_{N U} M_{J N}^{L U} P_{N}^{U}\right\rangle=B_{I J}^{K L} \sum_{N U} h_{I N J^{\prime}}^{K U}\left\langle M_{J N}^{L U} P_{J^{\prime}}^{V}\right\rangle .
$$

Similarly the expression

$$
\left\langle(M A)^{T} B_{I J}^{K L} P_{J}^{L}\right\rangle
$$

that stems from (75) can be transformed into

$$
B_{I J}^{K L} \sum_{I^{\prime}, V^{\prime}}\left\langle M_{I J^{\prime}}^{K V^{\prime}} P_{J^{\prime \prime}}^{W}\right\rangle h_{J^{\prime} J J^{\prime \prime}}^{V^{\prime} L W}
$$

Collecting all expressions and choosing only one matrix element $B$ with its specific indices, we obtain our final result

$$
G_{I J}^{K L}=h_{I, I^{\prime}, I^{\prime \prime \prime}}^{K L V} \sum_{J W}\left\langle M_{I^{\prime \prime} J}^{V W} P_{J}^{W}\right\rangle-\sum_{N U} h_{I N J^{\prime}}^{K U V}\left\langle M_{J N}^{L U} P_{J^{\prime}}^{V}\right\rangle-\sum_{J^{\prime}, V^{\prime}} h_{J^{\prime} J J^{\prime \prime}}^{V^{\prime} L}\left\langle M_{I J^{\prime}}^{K V^{\prime}} P_{J^{\prime \prime}}^{W}\right\rangle .
$$

This is the desired extension of the theorem of section 4 to a composite quantum system.

\section{Conclusion and outlook}

Some general remarks about the applicability of our above formalism may be in order. The projection operators correspond, at least in general, to physical observables, such as occupation numbers, (complex) amplitudes etc. Using them, we may calculate correlation functions of the form

$$
\left\langle P_{I, J}^{V}(t) P_{I^{\prime}, J^{\prime}}^{V^{\prime}}\left(t^{\prime}\right)\right\rangle
$$

or

$$
\left\langle\left(P_{I, J}^{V}(t)-\left\langle P_{I, J}^{V}(t)\right\rangle\right)\left(P_{I^{\prime}, J^{\prime}}^{V^{\prime}}\left(t^{\prime}\right)-\left\langle P_{I^{\prime}, J^{\prime}}^{V^{\prime}}\left(t^{\prime}\right)\right\rangle\right)\right\rangle .
$$

In particular, the latter form (83) enables us to determine the contribution of the fluctuations. We may thus determine the error made by a quantum device, e.g. by a logical gate. The formalism is rather general in that it does not only apply to elementary excitations, but also to general collective states provided that they can be characterized by quantum numbers and that their generalized Heisenberg equations are known.

A final remark should be made. As our above formalism reveals, Hamiltonian quantum systems are noise free. Only when they are coupled to reservoirs that cause incoherent processes, fluctuations become manifest. 


\section{References}

1. Klimontovich Yu. L. Statistical Theory of Open Systems. Kluwer Academic Publishers, 1995

2. Haken H. Synergetic Computers and Cognition, $2^{\text {nd }}$ ed. Berlin, Springer, 2004, (to appear).

3. Haken H., Weidlich W. // Z. Physik, 1966, vol. 189, p. 1.

4. Haken H. Laser Theory. Encyclopedia of Physics XXV/2c. Berlin, Springer, 1970.

\section{Флуктуації в квантових пристроях}

\section{Г.Хакен}

Інститут теоретичної фізики,

Центр синергетики університету Штутгарта,

Пфаффенвальдрінг, 57/4, D-70550, Штутгарт, Німеччина

Отримано 16 березня 2004 р.

Логічні фільтри можна формалізувати за допомогою булевої алгебри, елементарні операції якої можна реалізувати в квантових пристроях, де використовується взаємодія макроскопічної кількості елементарних збуджень (електрони, дірки, фотони і т.д.). 3 ростом мініатюризації системи до нанорозмірів, стають важливими квантові флуктуації, і ними вже не можна нехтувати. На основі рівняння Гейзенберга для операторів народження і знищення елементарних збуджень визначено характеристики джерел шуму в складних квантових пристроях.

Ключові слова: квантові флуктуації, елементарні збудження, рівняння руху Гейзенберга, логічні пристрої, шуми в складних квантових системах

PACS: 05.40.-a, 05.30.-d 
